\title{
Le changement climatique concerne aussi la santé
}

\author{
Damiano Urbinelloa, Nino Künzlib \\ a Dr ès sciences, Office fédéral de la santé publique (OFSP), unité de direction Politique de la santé \\ b Prof. Dr med. et PhD, vice-directeur de I'Institut tropical et de santé publique suisse et directeur de la Swiss School of Public Health (SSPH+)
}

La revue médicale Lancet identifie le changement climatique comme la plus grande menace potentielle du XXI ${ }^{\mathrm{e}}$ siècle [1] ${ }^{*}$. Les changements climatiques se répercutent sur l'environnement, l'économie et la société et représentent, sur le plan global, un risque considérable pour la santé de la population. Une planification stratégique et la mise en œuvre de mesures d'adaptation au climat sont déjà aujourd'hui nécessaires pour faire face à ces défis. Le bénéfice pour la santé résultant des stratégies relatives aux conséquences du changement climatique sera le thème principal de la Conférence Suisse de Santé Publique.

Le climat va continuer de se modifier à long terme. D'après les modèles climatiques, on peut s'attendre jusqu'à la fin du XXI ${ }^{\mathrm{e}}$ siècle, suivant les scénarios et l'efficacité des mesures de politique climatique, à un réchauffement global allant jusqu'à $4,8^{\circ} \mathrm{C}$. Un grand nombre d'études prouvent que ces hausses de température s'accompagnent de risques pour la santé. Les évènements météorologiques extrêmes tels que les vagues de chaleur représentent déjà aujourd'hui un danger pour la santé. L'été 2003 nous l'a montré. Les prévisions climatiques indiquent par exemple qu'à l'avenir, les épisodes de forte chaleur seront plus fréquents et plus intenses.

La canicule de 2003 en Europe a été l'une des plus chaudes des cinq siècles passés. En Suisse et dans de nombreuses régions d'Europe centrale, les températures se situaient 3 à $5^{\circ} \mathrm{C}$ au-dessus de la moyenne à long terme [2]. Les estimations statistiques montrent qu'environ 70000 décès supplémentaires ont été enregistrés en Europe [3]. En Suisse, une mortalité supplémentaire de 975 personnes a été estimée, ce qui correspond à une part de $7 \%$ du taux de mortalité moyen pour les mois de juin à août 2003 [4].

La chaleur peut nettement entraver les capacités physiques et intellectuelles. Les répercussions directes de la chaleur sont par exemple le coup de chaleur, la déshydratation, la perte de connaissance, les crampes dues à la chaleur et l'hyperthermie [5]. Les périodes de forte chaleur représentent un risque mortel particulièrement pour les personnes âgées et les malades (notamment les personnes atteintes de maladies chro- niques) ainsi que les enfants en bas âge, car ces groupes vulnérables disposent d'une moins bonne régulation thermique. Ils transpirent moins et ressentent moins

Les pays à faible revenu ne disposent pas des ressources suffisantes pour effectuer des mesures de prévention ciblées.

la soif, ce qui peut influencer le cœur et le système circulatoire. Plusieurs études indiquent par ailleurs que les admissions dans les services d'urgence augmentent pendant les périodes de forte chaleur prolongées, principalement en raison de troubles respiratoires. Ainsi, les admissions dans les services d'urgence en raison de la chaleur ont augmenté d'environ 3\% dans les villes américaines [6]. Une situation semblable a également été observée dans les villes européennes [7]. Les températures élevées associées à l'absence de rafraîchissement nocturne et à une humidité élevée expliquent probablement ces résultats.

A la suite de la canicule de 2003, l'Office fédéral de la santé publique et l'Office fédéral de l'environnement ont élaboré des informations et des recommandations de comportement pour les personnes à risque, leurs proches, le personnel soignant et les médecins [8]. De plus, divers cantons ont implémenté des mesures de prévention telles que des systèmes d'alerte précoce pour assurer la santé de la population. L'exemple de la France montre que les stratégies de prévention peuvent endiguer avec succès les conséquences d'une vague de chaleur. Ainsi, la mortalité due à la chaleur a pu être nettement réduite durant la canicule de 2006 
grâce aux plans canicule intégrés. Sans ces mesures, des estimations ont montré que 6452 cas de décès supplémentaires auraient eu lieu, si l'on fait l'hypothèse que la mortalité supplémentaire liée à la canicule est similaire à 2003. Au final, 2065 décès dus à la chaleur ont été enregistrés. Grâce à ces mesures de prévention, on a donc pu éviter environ 4400 décès [9].

Le changement climatique influence aussi les mécanismes de transmission zoonotiques et par conséquent l'apparition de maladies infectieuses transmises par des vecteurs [10]. En conséquence des échanges commerciaux globaux, l'Aedes albopictus (moustique-tigre asiatique) s'est installé dans le sud de l'Europe où il s'adapte maintenant aux températures plus basses. Dans un contexte de changement des conditions climatiques et des augmentations de température prognostiquées, il faut s'attendre à une propagation de ce vecteur primaire responsable des fièvres de chikungunya et de dengue. Le chikungunya se caractérise par une forte fièvre, des myalgies, des exanthèmes et de fortes arthralgies, ce qui distingue le chikungunya de la fièvre de dengue [11]. Par ailleurs, on s'attend à ce que les étés caniculaires modifient la présence et la dynamique de dissémination des tiques et contribuent ainsi à une propagation des maladies transmises par les tiques que sont l'encéphalite à tique (MEVE) et la borréliose [12]. Dans les régions disposant d'un bon système de santé et d'une gestion de l'environnement efficace, le changement climatique n'aura par contre guère d'impact sur la présence de la malaria, autrefois indigène en Suisse. La leishmaniose transmise par les phlébotomes est endémique en Europe et présente un certain risque d'une propagation accrue en cas d'augmentation des températures. La leishmaniose peut se manifester par des lésions ulcéreuses sur la peau ou les muqueuses [11].

Il faut d'ailleurs souligner que l'apparition et la propagation des maladies infectieuses transmises par des vecteurs en Suisse ne sont pas en premier lieu imputables au changement climatique, mais plutôt au comportement de la population en matière de voyage.

Les cas de maladies infectieuses liées à l'alimentation et à l'eau augmentent également pendant les périodes de canicule. Un facteur déterminant pour l'apparition de telles maladies infectieuses est la manipulation inappropriée d'aliments ainsi que l'absence de mesures d'hygiène [13].

En outre, la hausse de la température moyenne peut impliquer un allongement de la saison des pollens et la possibilité d'apparition de nouveaux allergènes (par ex. les néophytes importés légalement et illégalement), ce qui constitue un problème de plus en plus important pour les personnes allergiques.

Les risques sanitaires liés à la chaleur ont des mécanismes complexes et sont influencés par d'innombrables facteurs (socioéconomiques, écologiques, etc.). Ils représentent un grand défi pour le système de santé. Le changement climatique n'est pas seulement un défi local, mais global. Les résultats publiés en 2014 dans le cinquième rapport d'évaluation de l'IPCC (Intergovernmental Panel on Climate Change) soulignent les importants défis mondiaux dans la politique climatique: les pays riches occidentaux, qui étaient les principaux responsables du changement climatique au cours des derniers siècles, sont de plus en plus en mesure d'endiguer les conséquences directes du changement climatique par des mesures locales. Les pays à faible revenu par contre ne sont pas seulement bien plus touchés par le changement climatique, mais ne disposent pas des ressources suffisantes pour mettre en œuvre des mesures de prévention ciblées. Les conséquences du changement climatique dans ces pays seront donc bien plus importantes que sous nos latitudes, ce qui s'accompagnera d'une augmentation de la pression migratoire vers les pays riches [14].

La Conférence Suisse de Santé Publique est cette année dédiée aux opportunités et aux risques du changement climatique et à son impact sur la santé de la population. Elle a pour thème principal «Public Health Benefits of Climate Change Policies». Des experts nationaux et internationaux présenteront et discuteront les possibles conséquences du changement climatique sur la santé ainsi que le bénéfice réciproque des mesures de protection du climat pour la santé de la population.

La Conférence Suisse de Santé Publique se déroulera les 17 et 18 septembre 2015 à l'Institut de Santé Globale de l'Université de Genève. Inscription: http://conference. public-health.ch/fr

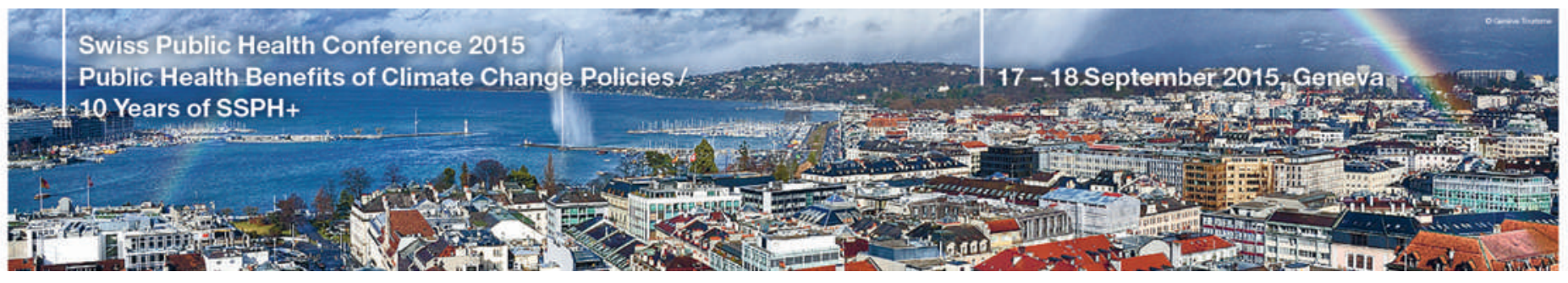




\section{Références}

1 Costello A, Abbas M, Allen A, Ball S, Bell S, Bellamy R, et al. Managing the health effects of climate change: Lancet and University College London Institute for Global Health Commission. Lancet. 2009;373:1693-733.

2 OcCC Beratendes Organ für Fragen der Klimaänderung. Hitzesommer 2003, Synthesebericht. Bern, November 2005.

3 Robine JM, Cheung SLK, Le Roy S, Van Oyen H, Griffiths C, Michel JP, et al. Death toll exceeded 70,000 in Europe during the summer of 2003. Comptes Rendus Biologies. 2008;331:171-8.

4 Grize L, Huss A, Thommen O, Schindler C, Braun-Fabrlander C. Heat wave 2003 and mortality in switzerland. Swiss Med Wkly. 2005;135:200-5

5 Thommen Dombois O, Braun-Fahrländer C. Gesundheitliche Auswirkungen der Klimaänderung mit Relevanz für die Schweiz. Literaturstudie im Auftrag der Bundesämter für Umwelt, Wald und Landschaft (BUWAL) und für Gesundheit (BAG). 2004.

6 Gronlund CJ, Zanobetti A, Schwartz JD, Wellenius GA, O'Neill MS. Heat, heat waves, and hospital admissions among the elderly in the United States, 1992-2006. Environ Health Perspect. 2014;122:1187-92.

7 Michelozzi P, Accetta G, De Sario M, D’Ippoliti D, Marino C, Baccin $M$, et al. High temperature and hospitalizations for cardiovascular and respiratory causes in 12 European cities. American Journal of Respiratory and Critical Care Medicine. 2009;179:383-9.

8 Bundesamt für Gesundheit (BAG). Informationsmaterial und Verhaltensempfehlungen verfügbar unter: www.hitzewelle.ch
9 Fouillet A, Rey G, Wagner V, Laaidi K, Empereur-Bissonnet P, Le Tertre A, et al. Has the impact of heat waves on mortality changed in France since the European heat wave of summer 2003 ? A study of the 2006 heat wave. Int J Epidemiol 2008;37(2):309-17.

10 ECDC (European Centre for Disease Prevention and Control). Climate change and communicable diseases in the EU Member States, Handbook for national vulnerability, impact and adaptation assessments. 2010.

11 Bassetti S. "Neue» Infektionskrankheiten in der Schweiz durch den Klimawandel? Schweiz Med Forum. 2009;9(50):905.

12 Gray JS, Dautel H, Estrada-Peña A, Kahl O, Lindgren E. Effects of Climate Change on Ticks and Tick-Borne Diseases in Europe. Interdiscip Perspect Infect Dis. 2009:593232.

13 Bless JP, Schmutz C, Suter K, Jost M, Hattendorf J, Mäusezahl Feuz $\mathrm{M}$ et al. A tradition and an epidemic: Determinants of the campylobacteriosis winter peak in Switzerland. European Journal of Epidemiology. 2014; 29(7):527-37.

14 IPCC, 2014: Climate Change 2014: Impacts, Adaptation, and Vulnerability. Part B: Regional Aspects. Contribution of Working Group II to the Fifth Assessment Report of the Intergovernmental Panel on Climate Change. Barros, V.R., C.B. Field, D.J. Dokken, M.D. Mastrandrea, K.J. Mach, T.E. Bilir, M. Chatterjee, K.L. Ebi, Y.O. Estrada, R.C. Genova, B. Girma, E.S. Kissel, A.N. Levy, S. MacCracken, P.R. Mastrandrea, and L.L. White (eds.). Cambridge University Press, Cambridge, United Kingdom and New York, NY, USA, $688 \mathrm{pp}$. 